\title{
Alberto Burgio, Le colpe di Rousseau. Letture, interpretazioni, fraintendimenti
}

\section{Franco Piva}

\section{Q OpenEdition}

1 Journals

\section{Edizione digitale}

URL: http://journals.openedition.org/studifrancesi/9561

DOI: 10.4000/studifrancesi.9561

ISSN: 2421-5856

\section{Editore}

Rosenberg \& Sellier

\section{Edizione cartacea}

Data di pubblicazione: 1 décembre 2007

Paginazione: 664-665

ISSN: 0039-2944

\section{Notizia bibliografica digitale}

Franco Piva, «Alberto Burgio, Le colpe di Rousseau. Letture, interpretazioni, fraintendimenti», Studi

Francesi [Online], 153 (LI | III) | 2007, online dal 30 novembre 2015, consultato il 13 janvier 2021. URL: http://journals.openedition.org/studifrancesi/9561 ; DOI: https://doi.org/10.4000/studifrancesi.9561

Questo documento è stato generato automaticamente il 13 janvier 2021.

\section{(c) $(1) \&$}

Studi Francesi è distribuita con Licenza Creative Commons Attribuzione - Non commerciale - Non opere derivate 4.0 Internazionale. 


\title{
Alberto Burgio, Le colpe di Rousseau. Letture, interpretazioni, fraintendimenti
}

\author{
Franco Piva
}

\section{NOTIZIA}

ALBERTO BURGIO, Le colpe di Rousseau. Letture, interpretazioni, fraintendimenti, «Dianoia.

Rivista di Storia della Filosofia», 11 (2006), pp. 167-203.

1 È nota la grande influenza che il pensiero di Rousseau ha esercitato sugli uomini e sulle idee che hanno guidato la Rivoluzione francese del 1789; meno nota è invece la «fortuna» di Rousseau nei decenni che seguirono quegli avvenimenti. Di fatto, soprattutto in Francia, si assistette a una vera e propria «derousseauizzazione», per riprendere la formula dell'autore di questo importante saggio. Molti di coloro che in un primo momento avevano parteggiato per Rousseau, se ne ritrassero poi in maniera netta; sulla scorta di fraintendimenti e interpretazioni che poco avevano a che fare osserva Burgio - con il pensiero del Ginevrino e molto invece con la reazione provocata dagli eccessi della Rivoluzione, che molti tesero a mettere sul conto delle idee che Rousseau avrebbe espresso nelle sue opere più note, in particolare nel Contrat social. Questo processo di «fraintendimento» iniziò presto in Francia ed ebbe tra i suoi primi $\mathrm{e}$ più importanti artefici Benjamin Constant che rimproverò a Rousseau di aver concepito la sovranità come un potere illimitato e di aver quindi conferito alla società «tutta intera [...] una sovranità senza limiti sui suoi membri», aprendo con ciò stesso una «strada infallibile verso il dispotismo». Questa interpretazione nasceva dal peso esercitato su Constant da una parte ben precisa del processo rivoluzionario che la Francia conobbe in quegli anni, ma essa, per la verità più complessa e articolata di quella che può suggerire la frase appena citata (come del resto Burgio mostra chiaramente nel suo studio), ha esercitato una forte influenza su tutta una parte degli 
storici e degli intellettuali francesi fin quasi alla fine dell'Ottocento con alcune significative propaggini anche nel secolo seguente.

Per trovare un'interpretazione più autentica del pensiero rousseauiano è necessario spostarsi in altri paesi, che meno della Francia hanno risentito della lettura negativa imposta da Constant e dal pensiero liberale sviluppatosi in quel paese; per esempio in Germania, dove fin dall'inizio l'opera di Rousseau fu letta e interpretata con maggiore distacco e, quindi, con maggiore aderenza alle intenzioni del suo autore; non sempre facili, per la verità, da enucleare nel loro significato più autentico. All'origine di questo sforzo ermeneutico si colloca Kant, la cui interpretazione risulta tuttavia più chiara alla luce di una lettura successiva: quella proposta negli anni Cinquanta del secolo scorso da un altro tedesco, Eric Weil. Per apprezzare appieno le ragioni di questo processo è necessario seguire passo passo A. Burgio nella sua analisi comparata delle interpretazioni proposte da Kant e da Weil. Quello che qui si può, e si deve sottolineare è la novità della lettura proposta da Burgio che, prendendo spunto per l'appunto dalla lettura di Weil e incrociandola con quella proposta alla fine del Settecento da Kant, fa notare come molti dei fraintendimenti su cui era caduto il pensiero liberale francese non solo non avevano, e non hanno alcuna ragione d'essere, ma possano risolversi in realtà in una lettura del pensiero rousseauiano molto più lineare e moderna di quanto fosse parso inizialmente possibile. Quello condotto da Burgio è quindi uno studio utile non solo per la ricezione di Rousseau nei due paesi presi in considerazione, ma anche per l'indicazione di un percorso ermeneutico capace di cogliere il vero pensiero di Rousseau su alcuni punti di fondamentale importanza anche per noi, oggi. 